\title{
Motion planning for parallel robots with non-holonomic joints
}

\author{
Krzysztof Tchoń, Janusz Jakubiak, Patrick Grosch and Federico Thomas
}

\begin{abstract}
Designing a robot manipulator with fewer actuators than the dimension of its configuration space - to reduce bulk, weight and cost- becomes feasible by introducing mechanical elements that lead to non-holonomic constraints. Unfortunately, the mechanical advantages of these non-holonomic designs are usually darkened by the complexity of their control. This paper deals with motion planning for parallel robots with non-holonomic joints shedding new light on their control strategies. As a case study, the motion planning problem is solved for a 3-ŬPU parallel robot, where $\breve{U}$ stands for a non-holonomic joint whose instantaneous kinematics are equivalent to that of a universal joint. It is thus shown how the three prismatic actuators can maneuver to reach any six-degree-of-freedom pose of the moving platform. The motion planning has been addressed as a control problem in the control system representation of the robot's kinematics and a motion planning algorithm has been devised based on a Jacobian inversion of the end-point map of the representation. Performance of the algorithm is illustrated with numeric computations.
\end{abstract}

Key words: parallel non-holonomic manipulator, Jacobian motion planning

\section{Introduction}

The joints of standard robots, either serial or parallel, implement lower kinematic pairs. An alternative to these joints are non-holonomic joints, a mechanical concept probably used for the first time in [8], which can be implemented using convex

Krzysztof Tchoń and Janusz Jakubiak

Institute of Computer Engineering, Control and Robotics, Wrocław University of Technology, Wrocław, Poland e-mail: krzysztof.tchon|janusz.jakubiak@pwr.wroc.pl

Patrick Grosch and Federico Thomas

Institut de Robòtica i Informàtica Industrial (CSIC-UPC), Barcelona, Spain e-mail: pgrosch|fthomas@iri.upc.edu 
bodies rolling on spherical surfaces. Two kinds of contacts have been considered: marble rolling, when the convex body can freely roll in contact with the sphere without slipping [4], and rubber rolling, when the convex body satisfies additionally a no-twist condition [9].

In the practical implementations of non-holonomic joints, the rolling convex body is usually a disk implementing a marble rolling contact with the sphere. If the disk rolls upon the interior surface of a spherical shell, the resulting joint is said to implement the Suslov constraint [15]. Alternatively, if the contact is performed on the outer surface of the spherical shell, the resulting joint is said to implement the Veselova constraint [3], the kind of non-holonomic joint used throughout this paper. Lower-mobility spatial parallel robots have become an active research topic in the field of parallel robot during the last decade because of their simple structure, low price and easy control. The dimension of the space of admissible velocities for the end-effector of this kind of parallel robots is lower than six and, if singular configurations are excluded, equal to the dimension of the tangent space of the reachable manifold. The substitution of a standard joint in a lower-mobility parallel robot by a non-holonomic joint with equivalent instantaneous kinematics has dramatic consequences: while the dimension of the space of admissible velocities for the endeffector remains the same, the dimension of the reachable space is increased. To the best of our knowledge, this idea was first used by Ben-Horin and Thomas in [1], where a three-legged parallel robot is proposed whose each leg is connected to the base through non-holonomic joints. The kinetostatics of this architecture was analyzed by Grosch et al. in [6], who proved that this robot was able to locally move its moving platform — excluding singular configurations - in a six-dimensional configuration space. In this paper we go a step further by presenting a solution to the motion planning problem for this robot which can be adapted to other designs.

It is worth to mention that the use of non-holonomic devices in the design of robot manipulators has some tradition. For example, in [13], Stammers et al. present a robot wrist that can attain any orientation with two motors only. This is achieved by means of a friction drive, using rollers on a spherical ball to which the end effector is fixed, and by fixing the two motors to the arm. In [12], Peshkin et al. present a passive spherical robot which can display programmable constraints. The device is based on a non-holonomic element involving a sphere and three reorientable rollers. In [11], Nakamura et al. describe an $n$-joint serial manipulator which can reach any pose in its $n$-dimensional configuration space with only two actuators. The joints of this manipulator are coupled by $(n-1)$ non-holonomic devices, based on spheres and rollers, so that its control is equivalent to maneuvering a car with $n$-trailers. Considerable effort has been made to clarify different aspects of non-holonomic mechanical systems [2]. A challenge in control of these systems results from a limited applicability of the feedback control, discovered by Brockett [5] and Lizárraga [10]. In this paper, the motion planning problem for the parallel non-holonomic robot will be addressed using the endogenous configuration space approach [14], specified in [7] to the class of mechanical systems including the parallel non-holonomic robot used as case study in this paper. The motion planning problem for the parallel non-holonomic robot will be decomposed into two steps: first the control system 
representing the robot's kinematics is subject to a feedback transformation, and afterwards the end-point map of the obtained system is inverted.

The remainder of the paper is organized as follows. Section 2 summarizes the main characteristics of the non-holonomic parallel robot used as the case study, and its instantaneous kinematics. Section 3 introduces the motion planning algorithm. Its performance is illustrated in section 4 by a numeric example. Section 5 concludes the paper.

\section{Instantaneous kinematics of the 3-ŬPU parallel robot}
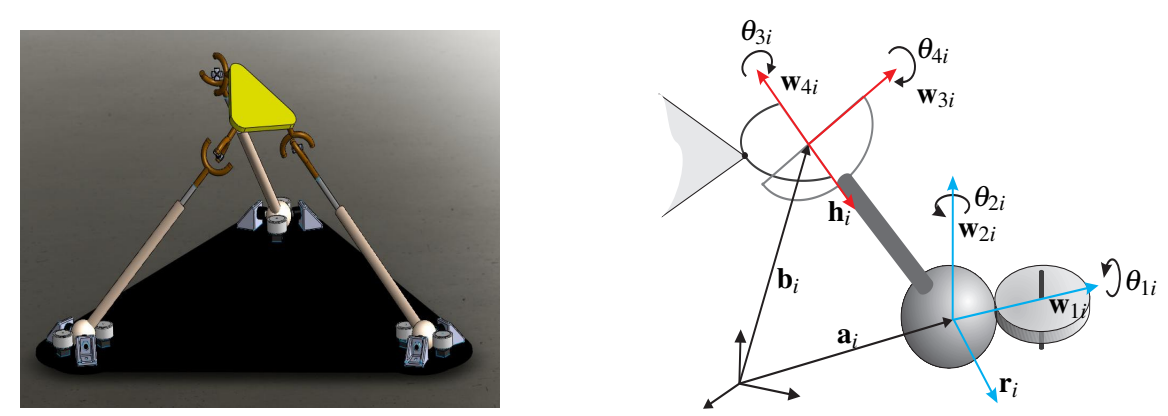

Fig. 1 The 3-ŬPU parallel robot used as a case study and notations associated with leg $i$

The parallel robot manipulator appearing in Fig. 1 (left) can be thought as a 3UPU lower mobility parallel robot in which each universal joint attached to the base is substituted by a non-holonomic joint (see [1] for details). Let us consider the leg number $i, i=1,2,3$. According to Fig. 1 , we shall introduce the following notations:

- $\mathbf{a}_{i}$ and $\mathbf{b}_{i}$ are the position vectors, in the base reference frame, of the centers of the sphere and of the universal joint, respectively.

- $l_{i}$ is the length of leg $i$, that is, $\left\|\mathbf{b}_{i}-\mathbf{a}_{i}\right\|$. Then, $\mathbf{g}_{i}=\left(\mathbf{b}_{i}-\mathbf{a}_{i}\right) / l_{i}$ is the unit vector in the direction of leg $i$.

- $\left\{\mathbf{w}_{1 i}, \mathbf{w}_{2 i}, \mathbf{r}_{i}\right\}$ defines a right-handed reference frame with origin at the center of the sphere. $\mathbf{w}_{1 i}$ is aligned with $\mathbf{a}_{i}$ and $\mathbf{w}_{2 i}$ is parallel to the roller axis.

- $\left\{\mathbf{w}_{3 i}, \mathbf{w}_{4 i}, \mathbf{h}_{i}\right\}$ defines a right-handed reference frame with origin at the center of the universal joint. $\mathbf{w}_{3 i}$ and $\mathbf{w}_{4 i}$ are defined by the two revolute axis constituting the universal joint.

- $\theta_{j i}$ is the joint variable denoting the rotation angle about the axis defined by $\mathbf{w}_{j i}$.

- $\mathbf{s}_{i}=\mathbf{h}_{i} \times \mathbf{r}_{i}-\left[\mathbf{g}_{i} \cdot\left(\mathbf{h}_{i} \times \mathbf{r}_{i}\right)\right] \mathbf{g}_{i}$ is the component of $\mathbf{h}_{i} \times \mathbf{r}_{i}$ perpendicular to $\mathbf{g}_{i}$.

Then, it can be proved that (see [6] for details):

$$
\left(\begin{array}{l}
\mathbf{1}_{3 \times 3} \\
\mathbf{0}_{3 \times 3}
\end{array}\right) \mathbf{i}=\left[\begin{array}{ll}
\mathbf{G}_{3 \times 3} & \mathbf{K}_{3 \times 3} \\
\mathbf{S}_{3 \times 3} & \mathbf{J}_{3 \times 3}
\end{array}\right]\left(\begin{array}{c}
\dot{\mathbf{p}} \\
\omega
\end{array}\right),
$$


where $\mathbf{i}=\left(\dot{l}_{1}, \dot{l}_{2}, \dot{l}_{3}\right)^{T}$ is the vector of velocities in the actuators, $\left(\begin{array}{c}\dot{\mathbf{p}} \\ \omega\end{array}\right)$ is the vector of linear and angular velocities of the moving platform, $\mathbf{1}_{3 \times 3}$ and $\mathbf{0}_{3 \times 3}$ are the $3 \times$ 3 identity and the zero matrix, respectively, and $\mathbf{G}=\mathbf{G}(\mathbf{p}, \mathbf{R}), \mathbf{K}=\mathbf{K}(\mathbf{p}, \mathbf{R}), \mathbf{S}=$ $\mathbf{S}(\mathbf{p}, \mathbf{R}), \mathbf{J}=\mathbf{J}(\mathbf{p}, \mathbf{R})$ are $3 \times 3$ matrices dependent on the end-effector pose (position and orientation) $(\mathbf{p}, \mathbf{R}) \in \mathbb{R}^{\mathbf{3}} \times \mathbf{S O}(\mathbf{3})$ whose entries are defined as

$$
\begin{array}{ll}
\mathbf{K}^{T}[i,:]=\left(\mathbf{b}_{i}-\mathbf{p}\right) \times \mathbf{g}_{i}, & \mathbf{G}^{T}[i,:]=\mathbf{g}_{i} \\
\mathbf{J}^{T}[i,:]=\left(\mathbf{b}_{i}-\mathbf{p}\right) \times \mathbf{s}_{i}-l_{i}\left(\mathbf{r}_{i} \cdot \mathbf{g}_{i}\right) \mathbf{h}_{i}, & \mathbf{S}^{T}[i,:]=\mathbf{s}_{i},
\end{array}
$$

where $\mathbf{A}[i,:]$ denotes the $i$-th row of a matrix $\mathbf{A}$.

\section{Motion planning}

As a starting point we shall adopt the kinematics representation (1) of the parallel non-holonomic robot. Assuming invertibility of the whole block matrix standing on the right hand side of (1) and taking $\mathbf{u}=\mathbf{i}$ as a control variable, the kinematics model is converted to the driftless control system

$$
\dot{\mathbf{p}}=\mathbf{E}(\mathbf{p}, \mathbf{R}) \mathbf{u}, \quad \dot{\mathbf{R}}=[\mathbf{F}(\mathbf{p}, \mathbf{R}) \mathbf{u}] \mathbf{R}
$$

used in [7], where [ ] $: \mathbb{R}^{3} \rightarrow$ so(3) denotes the standard Lie algebras isomorphism of $\mathbb{R}^{3}$ with the cross product and the space of skew symmetric $3 \times 3$ matrices with the matrix commutator, so that $[\mathbf{v} \times \mathbf{w}]=[\mathbf{v}][\mathbf{w}]-[\mathbf{w}][\mathbf{v}]$, and

$$
\left[\begin{array}{l}
\mathbf{E}(\mathbf{p}, \mathbf{R}) \\
\mathbf{F}(\mathbf{p}, \mathbf{R})
\end{array}\right]=\left.\left[\begin{array}{ll}
\mathbf{G} & \mathbf{K} \\
\mathbf{S} & \mathbf{J}
\end{array}\right]^{-1}\right|_{3 \text { first columns }}
$$

Given the control system (3), the motion planning problem for the parallel nonholonomic robot can be stated in the following way: compute a control function $\mathbf{u}(t)$ steering the system from an initial end effector pose $\left(\mathbf{p}_{0}, \mathbf{R}_{0}\right)$ to the desired one $\left(\mathbf{p}_{d}, \mathbf{R}_{d}\right)$ within a prescribed time $T$. More formally, setting $\mathbf{p}(t)=\mathbf{p}_{\mathbf{p}_{0}, \mathbf{R}_{0}, t}(\mathbf{u}(\cdot))$, $\mathbf{R}(t)=\mathbf{R}_{\mathbf{p}_{0}, \mathbf{R}_{0}, t}(\mathbf{u}(\cdot))$ to be the trajectory of (3) starting at $\left(\mathbf{p}_{\mathbf{0}}, \mathbf{R}_{\mathbf{0}}\right)$ and driven by the control $\mathbf{u}(t)$, this means that at time $T$ the end-point map of (3) assumes the prescribed values $\mathbf{p}(T)=\mathbf{p}_{d}$ and $\mathbf{R}(T)=\mathbf{R}_{d}$.

Due to the complexity of the matrix entries on the right hand side of (1), the analytic form of (3) is not very enlightening. To make it more tractable, two regularity assumptions will be made. First, the matrix $\mathbf{G}$ will be assumed invertible, resulting in the following form of the system (4)

$$
\left[\begin{array}{l}
\mathbf{E}(\mathbf{p}, \mathbf{R}) \\
\mathbf{F}(\mathbf{p}, \mathbf{R})
\end{array}\right]=\left[\begin{array}{c}
\mathbf{G}^{-1}+\mathbf{G}^{-1} \mathbf{K}\left(\mathbf{J}-\mathbf{S G}^{-1} \mathbf{K}\right)^{-1} \mathbf{S G}^{-1} \\
-\left(\mathbf{J}-\mathbf{S G}^{-1} \mathbf{K}\right)^{-1} \mathbf{S G}{ }^{-1}
\end{array}\right] .
$$

The second assumption is the invertibility of $\mathbf{S}$. Under this assumption the feedback 


$$
\mathbf{u}=\mathbf{G S}^{-\mathbf{1}}\left(\mathbf{J}-\mathbf{S G}^{-\mathbf{1}} \mathbf{K}\right) \mathbf{v}
$$

where $\mathbf{v} \in \mathbb{R}^{\mathbf{3}}$ is a new control, makes the control system (3) equivalent to

$$
\dot{\mathbf{p}}=\mathbf{S}^{-1} \mathbf{J v}, \quad \dot{\mathbf{R}}=-[\mathbf{v}] \mathbf{R} .
$$

Thanks to the regularity assumptions, the solution of the motion planning problem may be obtained in two steps: first a control $\mathbf{v}(t)$ solving the motion planning problem for the system (7) is found, and then the original control $\mathbf{u}(t)$ is computed using (6). The first step can be accomplished in accordance with the guidelines presented in [7], that will be concisely recalled below. Let $\mathbf{v}_{\theta}(t)$ be a family of control functions smoothly dependent on a parameter $\boldsymbol{\theta} \in \mathbb{R}$, and $\mathbf{p}_{t}(\boldsymbol{\theta})=\mathbf{p}_{\mathbf{p}_{0}, \mathbf{R}_{0}, t}\left(\mathbf{v}_{\boldsymbol{\theta}}(\cdot)\right)$, $\mathbf{R}_{t}(\boldsymbol{\theta})=\mathbf{R}_{\mathbf{p}_{0}, \mathbf{R}_{0}, t}\left(\mathbf{v}_{\theta}(\cdot)\right)$ denote the trajectory of the system (7) initialized at $\left(\mathbf{p}_{0}, \mathbf{R}_{0}\right)$ and subject to the control $\mathbf{v}_{\theta}(t)$. The derivation of the motion planning algorithm for the system (7) relies on an assumption that there exists a control family $\mathbf{v}_{\theta}(t)$, such that the error

$$
\left.\mathbf{e}(\boldsymbol{\theta})=\left(\mathbf{p}_{T}(\boldsymbol{\theta})-\mathbf{p}_{d}, \log \left(\mathbf{R}_{T}(\boldsymbol{\theta})\right) \mathbf{R}_{d}^{T}\right)\right),
$$

decreases to zero exponentially along with $\theta$ with a prescribed decay rate $\gamma>0$,

$$
\frac{d \mathbf{e}(\theta)}{d \theta}=-\gamma \mathbf{e}(\theta)
$$

The logarithm of the rotation matrix in (8) is defined as $\log \mathbf{R}=\frac{\alpha}{2 \sin \alpha}\left(\mathbf{R}-\mathbf{R}^{\mathbf{T}}\right)$, where $\cos \alpha=\frac{1}{2}(\operatorname{tr} \mathbf{R}-1)$ and the angle of rotation $0 \leq \alpha<\pi$.

To proceed, a pair of auxiliary variables will be introduced, denoted by $\mathbf{w}_{t}(\theta)$, $\mathbf{s}_{t}(\theta)$, satisfying the following dependencies

$$
\mathbf{w}_{t}(\theta)=\frac{\partial \mathbf{p}_{t}(\theta)}{\partial \theta}, \quad\left[\mathbf{s}_{t}(\theta)\right]=\frac{\partial \mathbf{R}_{t}(\theta)}{\partial \theta} \mathbf{R}_{t}^{T}(\theta)
$$

The differentiation with respect to $\theta$ of the matrices on the r.h.s of the system (7) results in a collection of differential equations (for details see [7], proof of Theorem 2.1)

$$
\left(\begin{array}{c}
\dot{\mathbf{w}}_{t}(\theta) \\
\dot{\mathbf{s}}_{t}(\boldsymbol{\theta})
\end{array}\right)=\left[\begin{array}{cc}
\mathbf{A}_{11 \theta}(t) & \mathbf{A}_{12 \theta}(t) \\
\mathbf{0} & -\left[\mathbf{v}_{\boldsymbol{\theta}}(t)\right]
\end{array}\right]\left(\begin{array}{c}
\mathbf{w}_{t}(\boldsymbol{\theta}) \\
\mathbf{s}_{t}(\boldsymbol{\theta})
\end{array}\right)+\left[\begin{array}{c}
\mathbf{B}_{1 \theta}(t) \\
-\mathbf{1}_{3}
\end{array}\right] \frac{d \mathbf{v}_{\theta}(t)}{d \boldsymbol{\theta}}
$$

where the entries of the matrices $\mathbf{A}_{11 \theta}(t), \mathbf{A}_{12 \theta}(t)$ and $\mathbf{B}_{1 \theta}(t)$ have been computed on the basis of the data provided in [6]. The assumption that $\mathbf{p}_{0}(\boldsymbol{\theta})=\mathbf{p}_{0}$ and $\mathbf{R}_{0}(\theta)=\mathbf{R}_{0}$ yields the initial conditions for $(11) \mathbf{w}_{0}(\boldsymbol{\theta})=\mathbf{0}$ and $\mathbf{s}_{0}(\boldsymbol{\theta})=\mathbf{0}$. With these initial conditions the solution of (11) at $T$ can be represented as

$$
\left(\begin{array}{c}
\mathbf{w}_{T}(\theta) \\
\mathbf{s}_{T}(\theta)
\end{array}\right)=\int_{0}^{T} \Phi_{\theta}(T, t) \mathbf{B}_{\theta}(t) \frac{d \mathbf{v}_{\theta}(t)}{d \theta} d t
$$

where the fundamental matrix $\Phi_{\theta}(T, t)$ satisfies the evolution equation $\frac{\partial \Phi_{\theta}(t, s)}{\partial t}=$ $\mathbf{A}_{\theta}(t) \Phi_{\theta}(t, s), \Phi_{\theta}(s, s)=\mathbf{1}_{6}$, and $\mathbf{A}_{\theta}(t)=\left[\begin{array}{cc}\mathbf{A}_{11 \theta}(t) & \mathbf{A}_{12 \theta}(t) \\ \mathbf{0} & -\left[\mathbf{v}_{\theta}(t)\right]\end{array}\right], \mathbf{B}_{\theta}(t)=\left[\begin{array}{c}\mathbf{B}_{1 \theta}(t) \\ -\mathbf{1}_{3}\end{array}\right]$. 
The integral operator in (12) can be regarded as a Jacobian operator of the parallel non-holonomic robot [14]. Now, it has been proved in [7] that the error vanishing formula (9) is tantamount to the integral equation

$$
\int_{0}^{T} \Phi_{\theta}(T, t) \mathbf{B}_{\theta}(t) \frac{d \mathbf{v}_{\theta}(t)}{d \theta} d t=-\gamma\left(\begin{array}{c}
\mathbf{p}_{T}(\theta)-\mathbf{p}_{d} \\
\mathbf{r}_{T}(\theta)
\end{array}\right)
$$

where $\left[\mathbf{r}_{T}(\boldsymbol{\theta})\right]=\log \left(\mathbf{R}_{T}(\boldsymbol{\theta}) \mathbf{R}_{d}^{T}\right)$. This being so, the motion planning algorithm for the parallel non-holonomic robot is obtained by solving the equation (13) using a generalized inverse of the Jacobian. If the Moore-Penrose pseudo inverse is chosen, the resulting differential equation for the control function $\mathbf{v}_{\theta}(t)$ takes the form

$$
\frac{d \mathbf{v}_{\theta}(t)}{d \theta}=-\gamma \mathbf{B}_{\theta}^{T}(t) \Phi_{\theta}^{T}(T, t) \mathbf{D}_{\theta}^{-1}\left(\begin{array}{c}
\mathbf{p}_{T}(\theta)-\mathbf{p}_{d} \\
\mathbf{r}_{T}(\boldsymbol{\theta})
\end{array}\right)
$$

The matrix $\mathbf{D}_{\theta}=\int_{0}^{T} \Phi_{\theta}(T, t) \mathbf{B}_{\theta}(t) \mathbf{B}_{\theta}^{T}(t) \Phi_{\theta}^{T}(T, t) d t$, is the Gram matrix of the system (11). Given the system (14), the solution of the motion planning problem is computed as the limit $\mathbf{v}(t)=\lim _{\theta \rightarrow+\infty} \mathbf{v}_{\theta}(t)$. The system (7) subject to the control $\mathbf{v}(t)$ produces a trajectory $(\mathbf{p}(t), \mathbf{R}(t))$. A suitable substitutions to the feedback equation (6) defines the control $\mathbf{u}(t)$ solving the motion planning problem for the parallel non-holonomic robot.

\section{Computations}

Since the motion planning algorithm (14) operates in an infinite dimensional space of control functions, its computer implementation needs to be preceded by the introduction of a finite dimensional space of controls. This is done in a standard way, by representing the control function by its truncated orthogonal expansion [14]. In this paper the truncated Fourier series is exploited, so each control $v_{i}(t), i=1,2,3$, will consist of a constant term and up to $h$ harmonics,

$$
v_{i}(t)=\lambda_{i, 0}+\sum_{k=1}^{h}\left(\lambda_{i, 2 k-1} \sin \frac{2 \pi}{T} k t+\lambda_{i, 2 k} \cos \frac{2 \pi}{T} k t\right)
$$

so the control is finitely parametrized by $\Lambda=\left(\lambda_{1,0}, \ldots, \lambda_{1,2 h}, \ldots, \lambda_{3,0}, \ldots, \lambda_{3,2 h}\right)^{T} \in$ $\mathbb{R}^{6 h+3}$. In the finite dimensional case the control family takes the form $\mathbf{v}_{\theta}(t)=$ $\mathbf{P}(t) \Lambda(\theta)$, where the block matrix $\mathbf{P}(t)$ aggregates the basic harmonic functions. Consequently, the differential equation (14) underlying the motion planning algorithm determines the control coefficients $\Lambda$

$$
\frac{d \Lambda_{\theta}}{d \theta}=-\gamma \mathbf{J}_{\mathbf{p}_{0}, \mathbf{R}_{0}, T}^{\#}\left(\Lambda_{\theta}\right)\left(\begin{array}{c}
\mathbf{p}_{T}(\theta)-\mathbf{p}_{d} \\
\mathbf{r}_{T}(\theta)
\end{array}\right)
$$



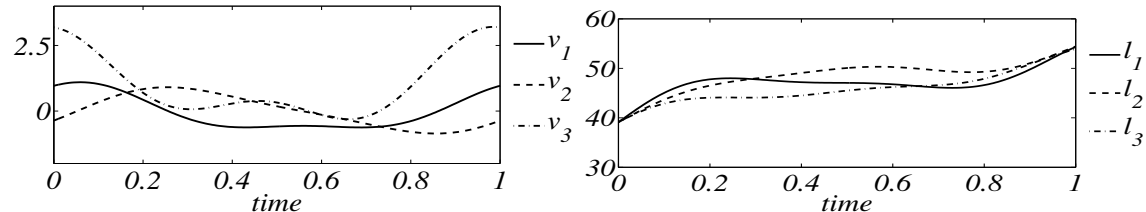

Fig. 2 Solution of the motion planning problem: controls $\mathbf{v}(t)$ and leg lengths $\mathbf{l}(t)$

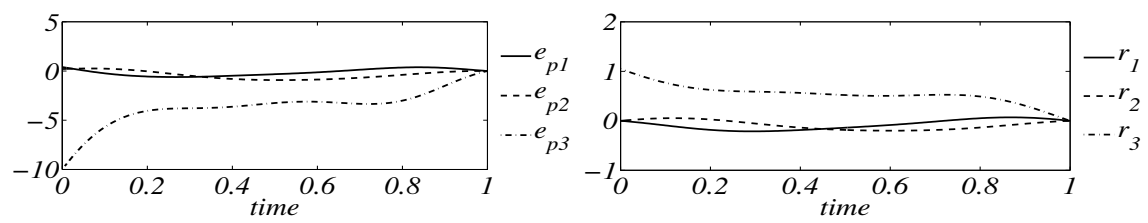

Fig. 3 Relative position $\mathbf{e}_{p}(t)$ and orientation $\mathbf{r}(t)$ trajectories

where $\mathbf{J}_{\mathbf{p}_{0}, \mathbf{R}_{0}, T}^{\#}\left(\Lambda_{\theta}\right)$ denotes the Moore-Penrose pseudo inverse of the $6 \times(6 h+3)$ Jacobian matrix

$$
\mathbf{J}_{\mathbf{p}_{0}, \mathbf{R}_{0}, T}\left(\Lambda_{\theta}\right)=\int_{0}^{T} \Phi_{\theta}(T, t) \mathbf{B}_{\theta}(t) \mathbf{P}(t) d t
$$

of the parallel non-holonomic robot. The differential equation (16) should be integrated numerically in accordance with a suitable integration scheme. In the sequel the simplest Euler scheme will be applied leading to the following difference equation for $\Lambda_{\theta}$, where $\theta=0,1, \ldots$

$$
\Lambda_{\theta+1}=\Lambda_{\theta}-\gamma \mathbf{J}_{\mathbf{p}_{0}, \mathbf{R}_{0}, T}^{\#}\left(\Lambda_{\theta}\right)\left(\begin{array}{c}
\mathbf{p}_{T}(\theta)-\mathbf{p}_{d} \\
\mathbf{r}_{T}(\theta)
\end{array}\right) .
$$

Performance of the motion planning algorithm will be illustrated with a numeric example. The initial position of the platform is $\mathbf{p}_{0}=(0,0,25)^{T}$, while its orientation $\mathbf{R}_{0}=R P Y(0,0,-\pi / 6)$ corresponds to the Roll-Pitch-Yaw angles $(0,0,-\pi / 6)$. The desired end effector position and orientation $\mathbf{p}_{d}=(-0.4,-0.2,35)^{T}$ and $\mathbf{R}_{d}=$ $R P Y(0,0,-\pi / 2)$. The initial values of control parameters have been set to 0 , except for $\lambda_{11}=\lambda_{21}=\lambda_{32}=0.5, \lambda_{30}=1$. The planning time horizon $T=1$. The algorithm has been stopped when the total error $\mathscr{E}(\theta)=\sqrt{\left\|\mathbf{p}_{T}(\theta)-\mathbf{p}_{d}\right\|^{2}+\left\|\mathbf{r}_{T}(\theta)\right\|^{2}}$ drops below $10^{-3}$. In the computations the number $h$ of harmonics is set to two. Results of computations are shown in figures 2 and 3. In Figure 3 the relative trajectories are shown, defined as $\mathbf{e}_{p}(t)=\mathbf{p}(t)-\mathbf{p}_{d}$ and $[\mathbf{r}(t)]=\log \left(\mathbf{R}(t) \mathbf{R}_{d}^{T}\right)$.

\section{Conclusion}

This paper provides a motion planning algorithm of the parallel non-holonomic robot. The algorithm's synthesis has been based on an application of the endoge- 
nous configuration space approach preceded by a feedback transformation of the system (3). Presented results provide a novel motion planning algorithm and essentially extend the applicability of the endogenous configuration space approach.

Acknowledgements The research of the first two authors was supported by a statutory grant from Wrocław University of Technology.

\section{References}

[1] Ben-Horin, P., Thomas, F.: Advances in Robot Kinematics, chap. A Nonholonomic 3-Motor Parallel Robot. Springer Verlag, Berlin (2008)

[2] Bloch, A.M.: Nonholonomic Mechanics and Control. Springer-Verlag, New York (2003)

[3] Borisov, A.P., Mamaev, I.S.: Non-holonomic Dynamical Systems, chap. Chaplygin's ball. The Suslov problem and Vaselova problem. Integrability and realization of constraints, pp. 118-130. Institute of Computer Science, Moscow (2002)

[4] Borisov, A.V., Mamaev, I.S.: The rolling body motion of a rigid body on a plane and a sphere. Hierarchy of dynamics. Regular and Chaotic Dynamics

[5] Brockett, R.W.: Differential Geometric Control Theory, chap. Asymptotic stability and feedback stabilization, pp. 171-181. Birkhäuser, Boston (1983)

[6] Grosch, P., Di Gregorio, R., Thomas, F.: Generation of under-actuated manipulators with non-holonomic joints from ordinary manipulators. ASME Journal Mech. Robotics 2(1) (2010)

[7] Jakubiak, J., Tchoń, K., Magiera, W.: Motion planning in velocity affine mechanical systems. Int. Journal of Control 83(9), 1965-1974 (2010)

[8] Kharlamov, A.P., Kharlamov, M.P.: Nonholonomic joint. Mekh. tverd. tela 27, $1-7$ (1995)

[9] Koiller, J., Ehlers, K.: Rubber rolling over a sphere. Regular and Chaotic Dynamics 12(2) (2007)

[10] Lizárraga, D.A.: Obstructions to the existence of universal stabilizers for smooth control systems. Math. Control, Signals, Syst. 16, 255-277 (2004)

[11] Nakamura, Y., Chung, W., Sordalen, O.J.: Design and control of the nonholonomic manipulator. IEEE Trans. Robot. Automat. 1(17), 48-59 (2001)

[12] Peshkin, M., Colgate, J.E., Moore, C.: Passive robots and haptic displays based on nonholonomic elements. In: Proc. 1996 IEEE ICRA, pp. 551-556 (1996)

[13] Stammers, C.W., Prest, P.H., Mobley, C.G.: A friction drive robot wrist: electronic and control requirements. Mechatronics 2(4), 391-401 (1992)

[14] Tchoń, K., Jakubiak, J.: Endogenous configuration space approach to mobile manipulators: a derivation and performance assessment of Jacobian inverse kinematics algorithms. Int. Journal of Control 76(9), 1387-1419 (2003)

[15] Vagner, V.: Geometrical interpretation of the motion of nonholonomic dynamical systems. Proc. Seminar on Vector and Tensor Analysis 5, 301-327 (1941) 